PROCEEDINGS OF THE

AMERICAN MATHEMATICAL SOCIETY

Volume 42, Number 1, January 1974

\title{
THE FIRST DERIVED EQUALISER OF A PAIR OF ABELIAN GROUP HOMOMORPHISMS
}

\author{
TIM PORTER
}

\begin{abstract}
In this note we attack the problem of classifying a pair of abelian group homomorphisms by introducing a construction which we call "the derived equaliser". After developing fairly simple methods for the calculation of these objects, we work an example in a simple case.
\end{abstract}

Introduction. The simplicity of the classification, up to isomorphism, of group homomorphisms by their kernels and cokernels tends to overshadow the problem for two or more homomorphisms. Recall that the First Isomorphism Theorem allows one to write any homomorphism as a composite of an epimorphism and an inclusion of a subgroup; the epimorphism is determined (up to isomorphism of group homomorphisms) by its kernel, and the inclusion can either be regarded as an invariant itself or it can be determined by its cokernel. The diagram

$$
G_{1} \stackrel{\theta}{\underset{\phi}{\longrightarrow}} G_{2}
$$

where $\theta$ and $\phi$ have isomorphic kernels and cokernels, may be very different indeed from the diagram,

$$
G_{1} \stackrel{\theta}{\underset{\theta}{\longrightarrow}} G_{2}
$$

where the two maps also have isomorphic kernels and cokernels.

A first approximation to a classification of such pairs of homomorphisms is via their "equaliser" or "difference kernel" [1] which measures how much they differ. The equaliser of $\theta$ and $\phi$ in (1) is the subgroup

$$
E(\theta, \phi)=\left\{g \in G_{1}: \theta(g)=\phi(g)\right\} .
$$

This is by no means a complete invariant, but in this paper we will investigate certain "derived invariants" of this for the case that $G_{1}$ and $G_{2}$ are abelian groups. We explicitly calculate the "first derived equaliser" $E^{1}(\theta, \phi)$ in a very simple case and produce a constructive description of it in general.

Received by the editors February 26, 1972.

AMS (MOS) subject classifications (1970). Primary 18G15, 20K30; Secondary $18 \mathrm{E} 25,20 \mathrm{~K} 40$.

(c) American Mathematical Society 1974 
1. Equalisers and derived equalisers. Let $\Sigma$ be the category with two objects, 1 and 2 , and with exactly two nonidentity morphisms as in the following diagram,

$$
1 \stackrel{\alpha}{\underset{\beta}{\longrightarrow}} 2
$$

Then a diagram of the form (1)

$$
G_{1} \underset{G(\beta)}{\stackrel{G(\alpha)}{\longrightarrow}} G_{2}
$$

where $G_{1}$ and $G_{2}$ are abelian groups, may be identified with a functor from $\Sigma$ to $A b$, the category of abelian groups. If $G: \Sigma \rightarrow A b$ and $F: \Sigma \rightarrow A b$ are two such functors, then a natural transformation $\phi$ from $F$ to $G$ gives precisely two homomorphisms $\phi_{1}: F_{1} \rightarrow G_{1}$ and $\phi_{2}: F_{2} \rightarrow G_{2}$ such that $\phi_{2} G(\alpha)=F(\alpha) \phi_{1}$ and $\phi_{2} G(\beta)=F(\beta) \phi_{1}$.

In particular, let $C(Z ; \Sigma)$ be the functor corresponding to the diagram

$$
Z \underset{1_{z}}{\stackrel{1_{z}}{\longrightarrow}} Z
$$

then a natural transformation $\phi$ from $C(Z, \Sigma)$ to $G$ gives a diagram

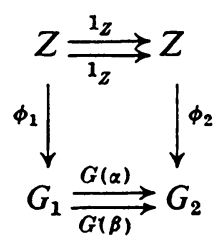

so $\phi_{2}(1)=G(\alpha) \phi_{1}(1)$ and also $\phi_{2}(1)=G(\beta) \phi_{1}(1)$. Thus $\phi_{1}(1)$ is an element of the equaliser $E(G(\alpha), G(\beta))$ and $\phi_{1}(1)$ completely determines $\phi$.

If we write $A b^{\Sigma}$ for the category of such functors and natural transformations we can identify

$$
\operatorname{Hom}_{A b} \Sigma(C(Z ; \Sigma), G) \cong E(G(\alpha), G(\beta)) \text {. }
$$

Since it is fairly easy to show that $A b^{\Sigma}$ has enough projectives (cf. [2]), it follows that we can form Ext functors in $A b^{\Sigma}$ using either of the usual methods (see [2]). Since the situation is fairly simple, we will use the more concrete representation as the set of congruence classes of exact sequences and define the first derived equaliser of $G(\alpha)$ and $G(\beta)$ to be the abelian group:

$$
E^{1}(G(\alpha), G(\beta))=\operatorname{Ext}_{A b}^{1} \simeq(C(Z ; \Sigma), G) .
$$

Higher derived equalisers could be defined, but are possibly zero.

2. A simple method of calculation of $E^{1}(G(\alpha), G(\beta))$. An element of $E^{1}(G(\alpha), G(\beta))$ may be represented as a congruence class of exact 
sequences. A typical exact sequence would be

$(A)$ :

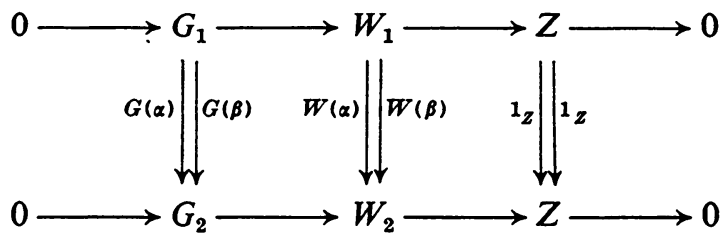

where the rows are exact and the $\alpha$ and $\beta$ half-diagrams commute separately. Since the rows are exact,

$$
W_{1} \cong G_{1} \oplus Z, \quad W_{2} \cong G_{2} \oplus Z,
$$

and since the $\alpha$ and $\beta$ half-diagrams commute,

and

$$
W(\alpha)(g, 0)=(G(\alpha) g, 0), \quad W(\beta)(g, 0)=(G(\beta) g, 0)
$$

$$
W(\alpha)(0,1)=(h(\alpha), 1), \quad W(\beta)(0,1)=(h(\beta), 1)
$$

for some $h(\alpha), h(\beta) \in G_{2}$. In fact $h(\alpha)$ and $h(\beta)$ completely determine the exact sequence $A$; we shall therefore write $A(h(\alpha), h(\beta))$ for this exact sequence. The problem of calculating $E^{1}(G(\alpha), G(\beta))$ thus reduces to determining for which $(h(\alpha), h(\beta))$ and $\left(h^{\prime}(\alpha), h^{\prime}(\beta)\right)$ the corresponding sequences are congruent. To this end we use the following lemma.

LEMMA.

$$
A(h(\alpha), h(\beta))+A(g(\alpha), g(\beta))=A(h(\alpha)+g(\alpha), h(\beta)+g(\beta))
$$

where addition is addition of exact sequences (as in Mac Lane [2]).

The proof is fairly easy and is given in full in [3]. The problem thus reduces to finding necessary and sufficient conditions so that $A(0,0) \equiv$ $A(h(\alpha), h(\beta))$, where $\equiv$ denotes congruence.

THEOREM. $A(h(\alpha), h(\beta)) \equiv A(0,0)$ if and only if there exist two elements $x_{1} \in G_{1}, x_{2} \in G_{2}$ such that $h(\alpha)=G(\alpha) x_{1}-x_{2}, h(\beta)=G(\beta) x_{1}-x_{2}$.

The proof is straightforward and again may be found in [3].

Corollary. $E^{1}(G(\alpha), G(\beta))$ is the quotient of $G_{2} \oplus G_{2}$ by elements of form $\left(G(\alpha) g_{1}-g_{2}, G(\beta) g_{1}-g_{2}\right)$ for $g_{1} \in \dot{G}_{1}, g_{2} \in G_{2}$.

3. A very simple case. Let $G_{1}=G_{2}=Z, G(\alpha)(1)=m, G(\beta)(1)=n$, then we have

$$
h(\alpha)=m x_{1}-x_{2}, \quad h(\beta)=n x_{1}-x_{2},
$$

i.e. $h(\alpha)-h(\beta)=(m-n) x_{1}$ implies $A(h(\alpha), h(\beta)) \equiv A(0,0)$. Thus $A(1,0)$ 
has period dividing $|m-n|$ and similarly for $A(0,1)$. However, $A(1,0)+$ $A(0,1)=A(1,1) \equiv A(0,0)$, so $A(0,1) \equiv(-1) A(1,0)$. It remains to find out if $A(r, 0) \equiv A(0,0)$ for any $r<|m-n|$.

Suppose $A(r, 0) \equiv A(0,0)$; then $h(\alpha)=r, h(\beta)=0$, so $m x_{1}-x_{2}=r$, $x_{2}=n x_{1}$ and $r=(m-n) x_{1}$, hence $A(1,0)$ has period exactly $|m-n|$; since $E^{1}(G(\alpha), G(\beta))$ is generated by $A(1,0), E^{1}(G(\alpha), G(\beta))=Z_{|m-n|}$.

Thus we have the following information in this case:

$$
\begin{gathered}
\operatorname{ker}(G(\alpha))=\operatorname{ker}(G(\beta))=0 ; \\
\operatorname{coker}(G(\alpha)) \cong Z_{|m|}, \quad \operatorname{coker}(G(\beta)) \cong Z_{|n|} ; \\
E(G(\alpha), G(\beta))=0 ; \quad C(G(\alpha), G(\beta)) \cong Z_{h},
\end{gathered}
$$

where $Z_{h}$ is the coequaliser of $G(\alpha)$ and $G(\beta)$, and $h$ is the highest common factor of $m$ and $n$, and

$$
E^{1}(G(\alpha), G(\beta))=Z_{|m-n|} \text {. }
$$

In this case these invariants allow $m$ and $n$ to be retrieved up to parity and the opposite system with $G^{\prime}(\alpha)=-m, G^{\prime}(\beta)=-n$ is plainly isomorphic to the one we have considered. Thus in this simple case the addition of $E^{1}$ to the invariants completely determines the system up to isomorphism.

\section{REFERENCES}

1. P. Freyd, Abelian categories. An introduction to the theory of functions, Harper's Series in Modern Math., Harper \& Row, New York, 1964. MR 29 \#3517.

2. S. Mac Lane, Homology, Die Grundlehren der Math. Wissenschaften, Band 114, Academic Press, New York; Springer-Verlag, Berlin, 1963. MR 28 \#122.

3. T. Porter, Generalised recurrence formulae and lim (1) (in preparation).

Department of Mathematics, Universtty College, Cork, Erre 